\title{
The Galactic positron annihilation radiation - an update based on recent SPI observations
}

\author{
Gerald Skinner* \\ University of Maryland, College Park, MD 20742, USA \\ \& CRESST / NASA-GSFC, Code 661, Greenbelt, MD 20771, USA \\ E-mail: skinner@milkyway.gsfc.nasa.gov
}

\section{Pierre Jean}

CESR, 7, av. du Colonel Roche, Cedex 4, Toulouse 31028, France

E-mail: jeandcesr.fr

\section{Jürgen Knödlseder}

CESR, 7, av. du Colonel Roche, Cedex 4, Toulouse 31028, France

E-mail: knodlseder@cesr.fr

\section{Pierrick Martin}

MPE, Postfach 1312, D-85741 Garching, Germany

E-mail: martinp@mpe.mpg.de

\section{Peter von Ballmoos}

CESR, 7, av. du Colonel Roche, Cedex 4, Toulouse 31028, France

E-mail: prbacesr.fr

\section{Georg Weidenspointner}

MPI Halbleiterlabor, Otto-Hahn-Ring 6, 81739 München, Germany

E-mail: georg.weidenspointner@hll.mpg.de

The origin of the positrons responsible for the $511 \mathrm{keV}$ annihilation radiation is a 40 year old mystery and one which has been a major objective for INTEGRAL since its inception. The signal being sought is small compared with the strong instrumental background line at $511 \mathrm{keV}$ and accurate modelling of that background line is a crucial aspect of the data analysis. Apparently inconsistent results have been reported using different approaches to background modelling.

We report on an analysis of all of the data available to us, including data obtained in a new 'pingpong' mode and on statistical assessments of different approaches to background subtraction. We conclude that there is indeed evidence for an asymmetry that has been reported between the emission from negative and positive longitudes but that this asymmetry is not best described by a simple imbalance in the disk emission, nor by an offset of the galactic bulge emission, but by a combination of the two. The apparent inconsistencies probably arise as a result of a combination of the differences between the background modelling approaches and a structure that is more complex than either of these two models suggests.

8th INTEGRAL Workshop, “The Restless Gamma-ray Universe”, - Integral2010,

September 27-30, 2010

Dublin Ireland

${ }^{*}$ Speaker. 


\section{Introduction}

Since its inception, a key objective for the Integral spectrometer, SPI, has been the study of the galactic $511 \mathrm{keV}$ line. Though the origin of the line is clearly the annihilation of positrons with electrons, the origin of the positrons themselves is still uncertain despite more than 40 years of work. The problem is not the lack of an explanation for their production, but a superfluity of possibilities and the difficulty of distinguishing between them and identifying which make significant contributions. The main clue available is the sky distribution of the line emission, though interpretation is complicated by the likelihood that the positrons travel appreciable distances before slowing down and annihilating, combined with uncertainties as to the extent and nature of the path.

Observations agree that the emission is strongly concentrated in a 'bulge' region $\left(\sim 10^{\circ}\right)$ around the galactic center. Although weak disk emission, $\sim 100^{\circ}$ in extent, is detectable, its surface brightness is remarkably low, implying an unexpectedly high bulge-to-disk ratio. Weidenspointner et al. [1] (here W08) reported an asymmetry in the emission from the inner disk, with that at negative galactic longitudes stronger by a factor of about 1.6 than that at positive longitudes, potentially providing important clues as to the origin of the positrons. In an independent analysis, Bouchet $e t$ $a l$. [2] (here B10) were unable to confirm this finding, seeing instead marginal evidence for an offset of the galactic bulge emission in the same direction. A major difference between the two analyses was the way the detector background was modelled and we here investigate how the background treatment can affect the conclusions.

\section{Background modeling}

Given the weakness of the astrophysical line and that the interest is in particular with regions of diffuse emission away from the relatively bright emission from around the galactic centre, accurate modeling of the background is essential. Two main approaches have been used in the past:

The detector pattern approach. While the backgrounds in each of the 19 SPI detectors vary with time, they do so such that there is a characteristic detector pattern that, to a good approximation, remains fixed in form and only a scaling factor changes. Separate patterns are used for the different instrument configurations with varying numbers of detectors functional and different numbers of annealings having taken place. The variation of the scaling factor with time can be taken into account by breaking the data into time intervals and fitting a different factor for each. In $B 10$ this method is used with intervals of $t_{F}=6$ hours. The patterns may be determined either from a subset of the instrument pointings identified as 'offs' as in B10, or as part of the overall fitting.

The tracer approach. The rate of saturating events in each detector ('Gedsat') is monitored and is found to be a remarkably good predictor of the $511 \mathrm{keV}$ line background (and that in other lines such as $1809 \mathrm{keV}$ ), particularly on relatively short timescales. To take account of longer term evolution of the background, W08 fit for each detector a scale factor each spacecraft orbit and a linear term accounting for long-term build up of activation. 


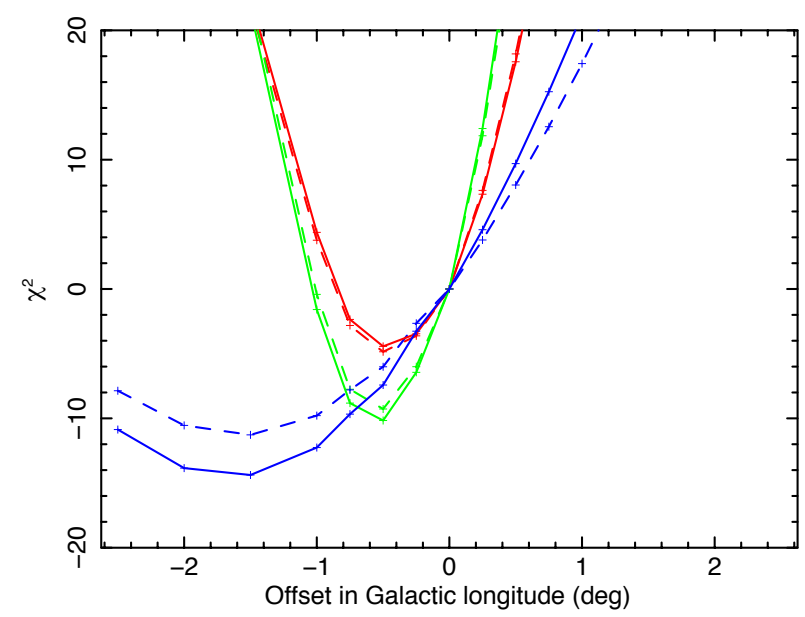

Figure 1: The variation of $\chi^{2}$ with the assumed offset in galactic longitude of either or both of two Gaussians representing the Galactic bulge. Green: both components displaced, Red: only narrow component displaced, Blue: only wide component displaced. Solid lines: tracer, dashed lines: detector pattern.

\section{Observations}

The data used here comprise all publicly available observations available as of 2010-Jul-06, plus observations made in AO6 and AO7 in a 'ping-pong' mode we proposed, designed to reduce the effects of systematic errors when comparing emission from negative and positive longitudes. Also included are observations to which we have data-rights specifically for this analysis. After cleaning to remove observations affected by solar flares, the total on-time is $122 \times 10^{6} \mathrm{~s}$ compared with $65 \times 10^{6} \mathrm{~s}$ in W08 and an estimated $103 \times 10^{6} \mathrm{~s}$ in $B 10^{1}$.

\section{Results}

In both W08 and B10 the galactic bulge is modelled by a combination of two Gaussian functions one narrow $\left(3.4^{\circ}\right.$ and $2.6^{\circ}$ FWHM respectively) and one wider $\left(11.6^{\circ}\right.$ and $\left.11^{\circ}\right)$. We used a model with a bulge component based on the latter of these two very similar descriptions and a disk, initially assumed to be symmetric, of the form adopted in W08. Fitting this model to the data using Maximum Likelihood, we confirm the findings of B10 that if both bulge components are offset together the best fit to the data is obtained with a galactic longitude offset of about $-0.5^{\circ}$ (1.e. towards negative longitudes), with a significance of just over $3 \sigma$. If instead the offset is assumed to be in the wider component alone, a somewhat better fit is obtained, with a $\sim 4 \sigma$ offset of $1.5^{\circ}$, whereas offsetting the narrow component alone leads to a worse fit (Fig. 1). Here the results using the tracer background model have been quoted, but essentially the same results are obtained with the detector pattern approach (Fig. 1, dashed line).

Next we consider the possibility of both an offset bulge and an inner $\left(|l|<50^{\circ}\right)$ disk asymmetry. Based on the above finding, we consider offsets of the wider bulge component only and

\footnotetext{
${ }^{1}$ It is assumed that the figure of $1.1 \times 10^{9} \mathrm{~s}$ quoted in that paper is a misprint.
} 


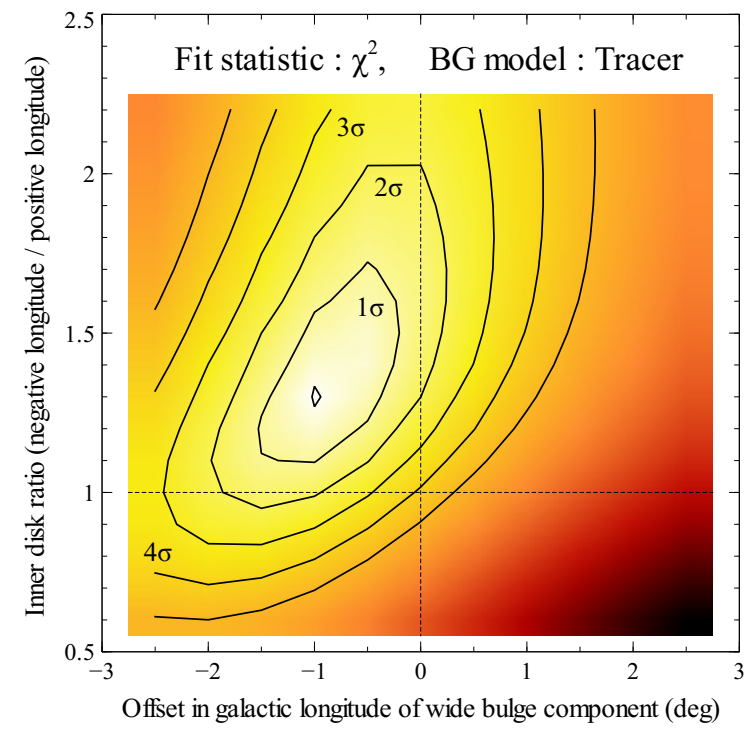

(a)

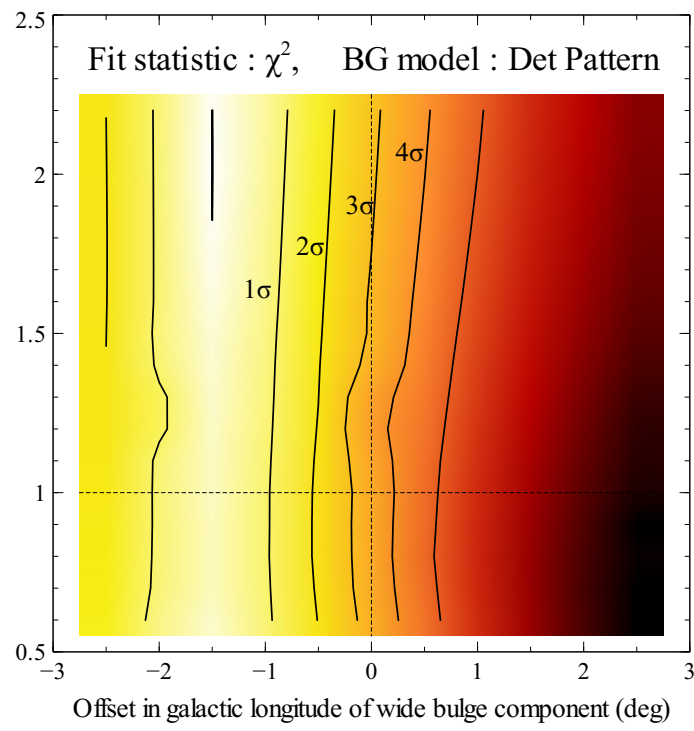

(b)

Figure 2: (a) The variation of $\chi^{2}$ with both the offset of the wide Gaussian bulge component and the disk ratio of the inner disk. The BG model used is the tracer one. (b) Ditto, but the BG model used is the detector pattern one.

combine this with a disk model in which the emission at negative longitudes is enhanced relative to that at positive longitudes by different factors. In Fig. 2(a) we show results obtained with the tracer approach. Considering points along the $\mathrm{X}$-axis (bulge offset), the minimum is at $-1.5^{\circ}$ as noted above, while along the Y-axis (asymmetry ratio) there is also a minimum at a ratio of $\sim 1.5$, consistent with W08. Among all of the models considered in the plot, the best description is with a combination of offset and asymmetry. The contours in Fig. 2(b) correspond to 1,2,.. $\sigma$ significance for 2 parameters of interest and consequently the significances are lower than for models with a single parameter. Nevertheless the symmetric case (offset $=0$, ratio $=1$ ) is excluded at more than $4 \sigma$.

If a similar analysis is performed using the detector pattern approach to background modelling, the results in Fig. 2(b) are obtained. Again there is the expected minimum along the X-axis at $\sim-1.5^{\circ}$, but this time, for a given offset, changing the disk ratio has little or no effect on the fit parameter. The method is largely insensitive to an asymmetry in the inner disk, explaining why the analysis in $B 10$ found no evidence for such an effect. This may be associated with the fact that the model includes a fitted parameter for the normalisation every $t_{F}=6$ hours. SPI's sensitivity to large-scale structures depends on differences between the flux observed in pointings that are well separated on the sky and large changes in pointing direction tend to occur only over timescales longer than this. An indication that this is indeed the explanation is found if the analysis is repeated with increased values of $t_{F}$. Although the fit becomes formally statistically unacceptable, the contours become more like those in Fig. 2(a).

\subsection{Comparison of background models}

Given the different results obtained with the two background modelling methods, it is important to consider which gives a better description of how the $511 \mathrm{keV}$ background in the SPI 
instrument behaves in practice. We have performed extensive statistical tests that are summarised briefly in Table 1. Note that as neither model is a subset of the other and only these two models are being compared, neither the F-test nor the AIC is formally applicable, though we believe that the F-test gives a strong indication in favour of the Tracer approach. Each of the other tests indicates that the tracer approach gives a somewhat better description of the instrument background than the Detector Pattern one. For these reasons we prefer to present results based on the Tracer background model.

Table 1: Tests of the goodness of fit with the two BG modelling approaches in an example case (see text). The $\chi^{2}$ values quoted use a variance based on the expected counts given the fitted model. More details will be provided in a publication under preparation.

\begin{tabular}{|c|c|c|}
\hline & \multicolumn{2}{|c|}{ Background modelling approach } \\
\hline & (a) Detector Pattern & (b) Tracers \\
\hline Observations, $(n)$ (detectors $\times$ pointings $)$ & 795165 & 795165 \\
\hline Parameters fitted, $n_{\text {dof }}$ (sky+background) & $3+6279$ & $3+12138$ \\
\hline$\chi^{2}$ & 791063.5 & 782266.0 \\
\hline$\chi^{2}-n_{d o f}$ & 2180.5 & -755.0 \\
\hline Probability & 0.04 & 0.70 \\
\hline$\chi^{2} / n_{d o f}$ & 1.0028 & 0.9990 \\
\hline F-test & \multicolumn{2}{|c|}{ Tracer model preferred $\left(P<10^{-6}\right)$} \\
\hline Akaiki Information Criterion (AIC) & \multicolumn{2}{|c|}{ Detector pattern model preferred (not quantitative) } \\
\hline Wald-Wolfowitz run test & $\begin{array}{c}399052 \\
397354 \pm 446 \\
3.81 \sigma\end{array}$ & $\begin{array}{c}397160 \\
397368 \pm 446 \\
-0.47 \sigma\end{array}$ \\
\hline
\end{tabular}

\subsection{Ping-pong observations}

Following observing proposals we made in response to AO-6 and AO-7, observations have been made in a special 'ping-pong' mode designed to minimize systematic errors in comparing the $511 \mathrm{keV}$ flux from positive and negative longitudes. Dithering patterns of 25 pointings were performed, alternately centred in the galactic plane at $l=+25^{\circ}$ and at $l=-25^{\circ}$ as indicated in Fig. 3(a). Often it was possible to obtain 2 scan patterns in each region within the same orbit. In this way the dependence of results on background modelling is minimised. Fig. 3(b) shows that a $511 \mathrm{keV}$ excess is seen in the negative longitude observations by forming a difference spectrum that is independent of background modelling. Because of detector failures, an observing time that was less than anticipated, and an increasing background due to the solar cycle, the significance is still relatively low, but more data are being accumulated.

\subsection{Locating the excess}

In order to explore the nature of the apparent excess in $511 \mathrm{keV}$ emission at negative longitudes we have fitted the data with a model with bulge emission and a symmetrical disk plus a point source placed at trial positions spread over a grid of latitude and longitude positions. The bulge 
a)

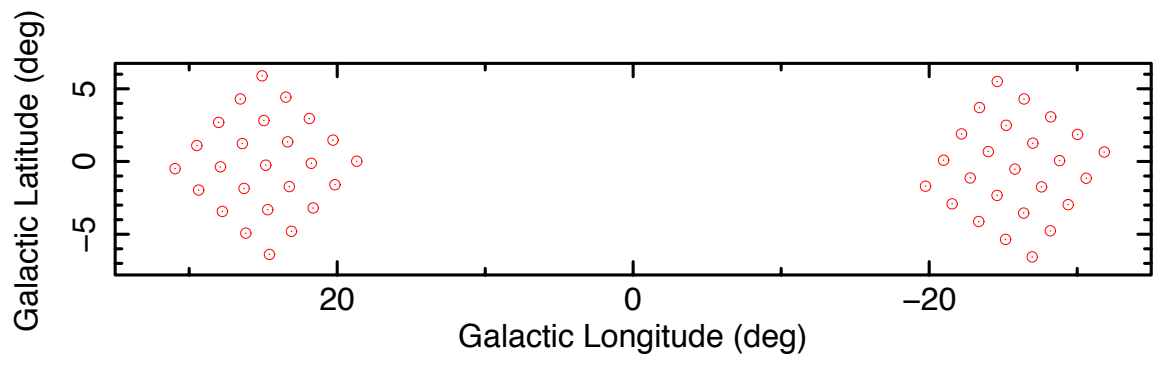

b)

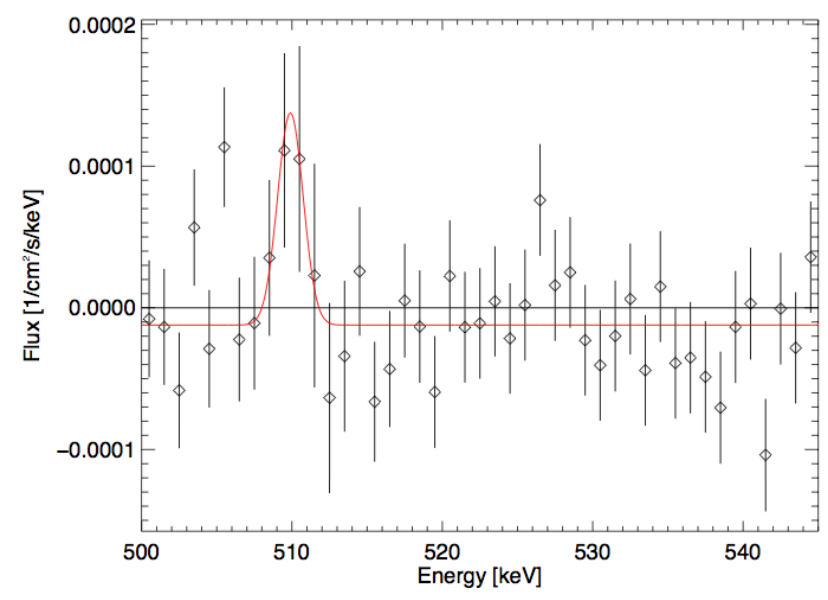

Figure 3: (a) A typical sequence of pointings for the 'ping-pong' observations. (b) A difference spectrum formed from the mean count rate in the negative longitude region, less that in the positive longitude one.

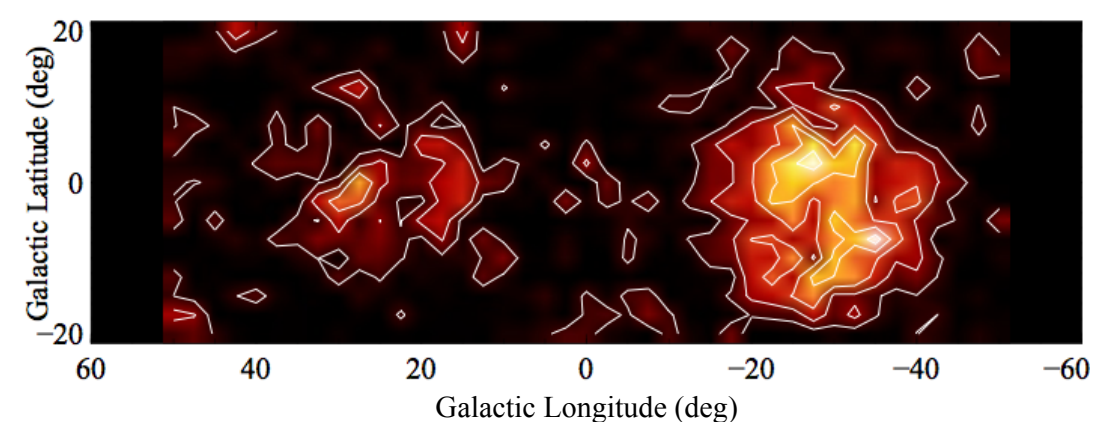

Figure 4: Adding a point source to a model with an offset bulge and symmetrical disc. The significance of the added source is shown as a function of its assumed position. Contours are at 1, 2, 3, 4, $5 \sigma$. Background model: Tracer.

was represented as narrow and wide Gaussians, with the wide component offset to $l=-1.5^{\circ}$ and the disk model was a symmetric form of that used in W08. The improvement in $\chi^{2}$ resulting from the addition of the point source is shown in Fig. 4. The low levels in the centre of the map show that the emission from the bulge region is apparently well accounted for by the narrow plus offset-wide Gaussians, but there is strong evidence for an additional excess, not described by the offset bulge, at $-20^{\circ}$ to $-40^{\circ}$. 


\section{Conclusions}

The evidence for an asymmetry in the $511 \mathrm{keV}$ emission seems strong, though the form that it takes is less clear. To some extent the conclusions depend on how the background modelling is done. We have argued that of the two approaches considered, the tracer one gives a statistically more satisfactory description. Using that approach, within the range of models considered for the asymmetry in the sky emission - a (wider) bulge component offset in galactic longitude and/or a 'split' disk with different scaling factors for negative and positive longitude - a combination of both effects is preferred, though either alone is not strongly excluded.

This paper is a preliminary report on an extensive series of investigations; a detailed publication is in preparation.

\section{References}

[1] G. Weidenspointner, Georg et al., An asymmetric distribution of positrons in the Galactic disk revealed by gamma-rays, Nature 451 (2008) 159 (W08)

[2] L.Bouchet, J.-P. Roques, E. Jourdain, On the Morphology of the Electron-Positron Annihilation Emission as Seen by Spi/Integral ApJ 72020101772 (B10) .... 